\title{
A Canine Model to Assess the Biochemical Stress Response to Laparoscopic and Open Surgery
}

\author{
ROBERT MARCOVICH, M.D., ANTOINETTE L. WILLIAMS, B.S., BRIAN D. SEIFMAN, M.D., \\ and J. STUART WOLF, JR., M.D.
}

\begin{abstract}
Purpose: To develop an animal model to assess the stress response to open and laparoscopic surgery. Such a model would allow objective physiologic assessment of the putative benefits of laparoscopy and provide a framework in which to compare modifications in operative and anesthetic technique that might decrease the stress of surgery.

Materials and Methods: Mongrel dogs underwent laparoscopic $(\mathrm{N}=12)$ or open surgical $(\mathrm{N}=12)$ left nephrectomy. In 11 control animals, after induction of anesthesia and line placement, the animal underwent either no intervention (open surgery sham; $\mathbf{N}=6$ ) or pneumoperitoneum only (laparoscopic sham; $\mathbf{N}=\mathbf{5}$ ). Serum glucose and cortisol were measured preoperatively, at skin closure, and at 4, 8, and 24 hours postoperatively. Values at each time point were compared in the laparoscopic and open surgical nephrectomy groups and in each of the two nephrectomy groups and their respective shams.

Results: Compared with baseline, there was a sharp rise in serum cortisol at the time of skin closure, with a gradual decline to baseline values by 24 hours, in all experimental animals. Significantly lower serum cortisol concentrations were seen at 4 and 8 hours postoperatively in the laparoscopic group than in the open surgery group. Cortisol was significantly higher in the open group than in the sham-open group at all time points, whereas cortisol was greater in the laparoscopic group than in the pneumoperitoneum-only group only at the 4-hour time point. No differences were seen in serum glucose between groups.

Conclusions: The serum cortisol concentration appears to be a good measure of surgical stress in the canine model. The rapid decline in serum cortisol after laparoscopy compared with open surgery may indicate a lesser degree, or quicker resolution, of surgical stress in the former. Furthermore, the similarity in cortisol curves between laparoscopy and pneumoperitoneum only suggests that surgical stress in laparoscopic surgery may be attributable mainly to the effects of pneumoperitoneum.
\end{abstract}

\section{INTRODUCTION}

$\mathbf{T}$ HE IMPLEMENTATION of laparoscopic and other minimally invasive surgical techniques in urology has been driven largely by the concept that these modalities result in less operative stress and postoperative pain than standard open surgery. Comparative studies have found that patients undergoing laparoscopic surgery tend to use less narcotic pain medication, have shorter hospital stays, and return to activity more quickly than patients undergoing analogous open procedures. ${ }^{1,2}$ However, studies of objective physiologic measures of surgical stress in humans subjected to laparoscopic and open surgery have not found great differences or have led to conflicting out- comes. $^{3-8}$ Additionally, newer surgical modalities, such as hand-assisted laparoscopy ${ }^{9}$ and minilaparoscopy, ${ }^{10}$ have been introduced, but objective assessment of their effects on surgical stress are lacking. A variety of pharmacologic interventions have been proposed to decrease postoperative pain, such as preemptive anesthesia ${ }^{11}$ or the use of capsaicin ${ }^{12}$ to temporarily desensitize local sensory nerves. However, we lack objective methods of measuring the effects of these and other surgical and anesthetic strategies.

Our goal, therefore, was to develop an animal model with which to reliably compare the effects of such maneuvers on the stress response. For this study, we chose to compare laparoscopic and open surgical nephrectomy in dogs. Our hypothesis

Department of Urology, University of Michigan and Veterans Affairs Medical Center, Ann Arbor, Michigan. 
Table 1. Anesthesia and Procedure Times

\begin{tabular}{lccc}
\hline & Mean time (min) & $( \pm S D)$ & P value \\
\hline & Open nephrectomy & Lap nephrectomy & \\
Anesthesia & $($ Group A) & (Group B) & 0.02 \\
Procedure & $111 \pm 24$ & $149 \pm 22$ & 0.0002 \\
& $57 \pm 15$ & $105 \pm 28$ & \\
& & & \\
Anesthesia & Open nephrectomy & Sham open surgery & NS \\
Procedure & $111 \pm 24$ & (Group C) \\
& $57 \pm 15$ & $96 \pm 15$ & \\
& & $52 \pm 12^{\mathrm{a}}$ & \\
Anesthesia & Lap nephrectomy & Pneumoperitoneum only & NS \\
Procedure & $149 \pm 22$ & $($ Group D) & NS \\
\hline
\end{tabular}

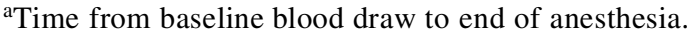

${ }^{\mathrm{b}}$ Pneumoperitoneum time.

was that postoperative biochemical measures, specifically the serum concentrations of cortisol and glucose, could be used to detect differences in the stress response to these rather invasive procedures.

\section{MATERIALS AND METHODS}

Animal studies were approved by the institutional review boards. Thirty-five male and female mongrel dogs weighing approximately 20 to $25 \mathrm{~kg}$ were fasted overnight and medicated with xylazine (2-4 mg/kg). Following anesthesiainduction with pentothal 20 to $30 \mathrm{mg} / \mathrm{kg}$ and endotracheal intubation, anesthesia was maintained with $1.5 \%$ halothane. An internal jugular venous catheter was placed to facilitate subsequent blood sampling.

The animals were divided into four groups. One group $(\mathrm{N}=$ 12) underwent open surgical left nephrectomy through a standardized 10-cm flank incision and another group of animals $(\mathrm{N}=12)$ laparoscopic left nephrectomy at a pneumoperitoneum pressure of $15 \mathrm{~mm} \mathrm{Hg}$ utilizing three ports (two $10 \mathrm{~mm}$ and one $5 \mathrm{~mm}$ ). Control animals consisted of six that had only general anesthesia and venous catheter placement (open-surgery sham) and five that underwent anesthesia, venous catheter placement, and pneumoperitoneum through a single $10-\mathrm{mm}$ port without any other surgical intervention (laparoscopic sham). We used a port for the latter, rather than a Veress needle alone, in order to effect complete evacuation of the insuf- flated $\mathrm{CO}_{2}$ at the end of the procedure. Anesthesia and procedure times of the control groups were matched with those of the experimental groups (Table 1). In the laparoscopic nephrectomy cohort, kidneys were removed through one of the $10-\mathrm{mm}$ ports after morcellation. In neither of the surgical groups was the adrenal gland excised during the procedure.

Blood was drawn from each subject at baseline (after induction of anesthesia and venous catheter placement) and then at skin closure; and at 4, 8, and 24 hours postoperatively. Blood was drawn through the indwelling external jugular line while an assistant gently held the animal still. No venipuncture, sedation, or artificial restraint was necessary. Samples were immediately centrifuged, and plasma was submitted for serum cortisol and glucose determination. Postoperative analgesia was not used.

\section{RESULTS}

Table 1 summarizes the anesthesia and operative times in the control and experimental animals. As would be expected, total intervention time (anesthesia plus operative) was significantly longer in the laparoscopic than in the open surgical group. Times were well matched in the open surgery and sham surgery groups and in the laparoscopic and pneumoperitoneum-only groups.

Table 2 shows the mean serum cortisol concentrationsat each time point for each of the four interventions. The skin closure, 4-hour, and 8-hour values were significantly different from baseline in the open nephrectomy group, while only the skin

Table 2. Mean Serum Cortisol $(\mu \mathrm{g} / \mathrm{dL} \pm \mathrm{SD})$

\begin{tabular}{lccccc}
\hline Procedure & Baseline & Skin closure & 4 hour & 8 hour & 24 hour \\
\hline Lap. nephrectomy & $1.6 \pm 1.8$ & $13.6 \pm 1.9^{\mathrm{a}}$ & $5.6 \pm 3.1^{\mathrm{a}}$ & $2.6 \pm 1.6$ & $2.2 \pm 0.8$ \\
Open surgery & $2.2 \pm 1.7$ & $10.5 \pm 2.9^{\mathrm{a}}$ & $12.4 \pm 8.4^{\mathrm{a}}$ & $6.8 \pm 3.6^{\mathrm{b}}$ & $2.6 \pm 1.7$ \\
Open sham & $4.6 \pm 4.5$ & $7.3 \pm 6.0$ & $2.5 \pm 1.7$ & $1.4 \pm 0.8$ & $2.5 \pm 1.8$ \\
Pneumoperitoneum only & $3.9 \pm 3.8$ & $15.6 \pm 3.9^{\mathrm{a}}$ & $2.4 \pm 1.2$ & $1.8 \pm 1.0$ & $2.7 \pm 3.8$ \\
\hline
\end{tabular}

${ }^{\mathrm{a}}$ Higher than baseline ( $p<0.0001$; ANOVA with multiple comparisons).

${ }^{\mathrm{b}}$ Higher than baseline ( $p=0.01$; ANOVA with multiple comparisons). 
closure and 4-hour values were different from baseline in the laparoscopic group. Sham open surgery values were no different from baseline, while the level at skin closure was significantly elevated for the pneumoperitoneum-only cohort.

Figure 1 graphically demonstrates the change from baseline of serum cortisol concentrations at skin closure and at 4, 8, and 24 hours postoperatively in open $v$ laparoscopic nephrectomy (panel A), open $v$ sham surgery (panel B), and laparoscopic nephrectomy $v$ pneumoperitoneum only (panel C).

In summary, serum cortisol concentrations peaked within 4 hours and fell to baseline by 24 hours in both of the experimental groups. Interestingly, cortisol concentrations were consistently higher at the time of skin closure in animals that underwent laparoscopic nephrectomy than in those that had open surgery; but overall, cortisol decreased significantly more rapidly in the laparoscopic group than in the open surgery group. In fact, cortisol continued to rise through the 4-hour span in the open surgery group but fell significantly by this time point in the laparoscopic cohort. By 8 hours, cortisol had come down to near-baseline concentrations in the laparoscopic group, while in the open surgery group, it remained significantly elevated. In the sham open-surgery group, serum cortisol did not differ significantly from baseline but was, as expected, significantly lower on average than in the animals undergoing open surgical nephrectomy. Cortisol concentrations in the pneumoperitoneum-only group paralleled those in the laparoscopic nephrectomy group, except at 4 hours, where the laparoscopic group had significantly higher values.

Finally, serum glucose concentrations showed no significant pattern of variability among either of the experimental or control groups (data not shown).

\section{DISCUSSION}

In 1959, Egdahl ${ }^{13}$ demonstrated that the endocrine response to surgical trauma, as represented by an acute elevation in the adrenal vein corticosteroid concentration, is initiated by transmission of neural impulses via sensory afferents from the site of injury to the central nervous system. He also showed that denervation of the injured tissue prior to the insult results in a lack of corticosteroidrelease. Such denervation obviously leads to anesthesia of the injured part. The dependence of both the endocrine response to injury and the perception of pain on similar intact afferent neural pathways has led to the concept that pain may be quantified in a more or less objective fashion by measurement of the magnitude of the neuroendocrineresponse.

This idea becomes especially valuable when attempting to measure pain in animals. Studies have pointed out significant variability among observers attempting to visually assess and numerically rate pain in postsurgical dogs, ${ }^{14}$ as well as a lack of correlation of observers' subjective measures of pain (such as visual analog scores) with commonly accepted objective measures of pain in animals, such as heart rate, breathing rate, and blood pressure. ${ }^{15}$ Thus, in this study of operative stress, we chose to measure serum cortisol, rather than rely on potentially inaccurate subjective assessments. We also limited our investigation to measuring the neuroendocrine response, which may better serve as a surrogate for pain assessment than the cytokines (interleukins 1 and 6; tumor necrosis factor), which are
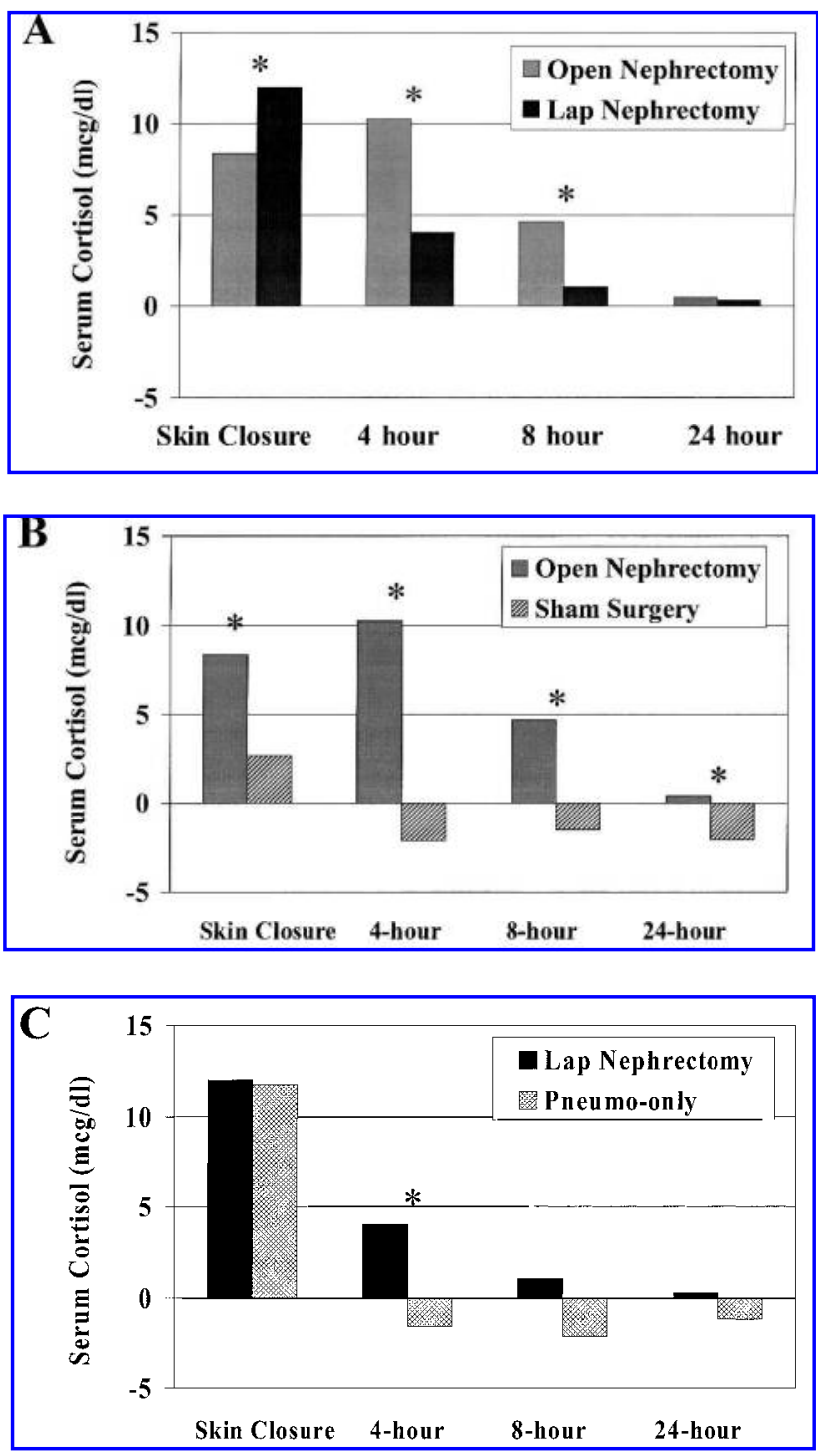

FIG. 1. Change from baseline of serum cortisol concentrations at skin closure, 4 hours, 8 hours, and 24 hours in (A) open $v$ laparoscopic nephrectomy; (B) open $v$ sham surgery; and (C) laparoscopic nephrectomy $v$ pneumoperitoneum only $(* P<0.05)$.

released at the site of injury and better serve as markers of the extent of tissue injury. ${ }^{16}$

Prior studies comparing the stress response to open and laparoscopic surgery in human subjects have resulted in conflicting outcomes. The majority of these studies were in patients undergoing cholecystectomy, $3,4,6,8$ appendectomy, 5 or abdominal hysterectomy. In the first group, Karayiannakis and associates $^{3}$ and Yoshida and colleagues ${ }^{6}$ found postoperative serum cortisol to be significantly higher in patients who had undergone open cholecystectomy than in patients who had had laparoscopic cholecystectomy. However, Jakeways and cowork$\mathrm{ers}^{8}$ and Ortega et $\mathrm{al}^{4}$ found no significant difference in similar investigations. Bozkurt and colleagues ${ }^{5}$ found no difference in cortisol concentrations, or any other postoperative biochemical measure, in 23 children who were explored either laparoscop- 
ically or via laparotomy for acute abdominal pain. This study may have been flawed in that the neuroendocrine response was probably equally active in both groups preoperatively because of their common presentation with acute abdomen. Other possible reasons for the variability in outcomes of human studies such as these include the use of different anesthetic and sedative combinations, which can differentially alter corticosteroid release ${ }^{16}$ small numbers of subjects; and predisposing patient factors that could have been controlled for by randomization. One significant potential source of confounding that cannot be avoided in any human study is the use of postoperative analgesia: opiates are known to suppress corticosteroid release, so those patients who use more narcotic pain medication (i.e., the ones undergoing open surgery) may blunt their cortisol response to a greater degree than those using less analgesia (the patients having laparoscopic procedures).

These considerations point to the necessity for controlled animal experimentation whenever one attempts to assess the physiologic impact of novel techniques or interventions on the operative stress response. In this study, we randomized dogs to open nephrectomy, laparoscopic nephrectomy, and sham open and laparoscopic procedures. No postoperative analgesia was used. Our results indicate an overall more rapid diminution in postoperative serum cortisol in those animals that underwent laparoscopy. We found that in the laparoscopic group, cortisol peaked at skin closure, while it continued to rise postoperatively in the open nephrectomy group. Our anesthetic regimen did not spuriously raise cortisol levels, and, interestingly, the cortisol curve for those animals who had had only a pneumoperitoneum mirrored the curve for the laparoscopic nephrectomy subjects. This might indicate that cortisol elevation in laparoscopy is attributable mainly to the effects of pneumoperitoneum rather than to the actual operative intervention. This finding is supported in the literature by the study of Ishizuka et al, ${ }^{17}$ who compared the stress response in women undergoing laparoscopy with $\mathrm{CO}_{2}$ pneumoperitoneum $v$ those undergoing laparoscopy with mechanical elevation of the abdominal wall. The $\mathrm{CO}_{2}$ insufflation was associated with a higher intraoperative serum cortisol concentration than was mechanical abdominal wall elevation. It is unknown whether the effect of pneumoperitoneum on serum cortisol is secondary to a noxious stimulus or is an indirect result of hypercarbia, a known stimulant of cortisol release. ${ }^{18}$

\section{CONCLUSION}

Serum cortisol appears to be a good measure of surgical stress in the canine model. The rapid decline in serum cortisol after laparoscopy compared with open surgery may indicate a lesser degree, or quicker resolution, of surgical stress in the former. Our data suggest that pneumoperitoneum, rather than the actual surgical intervention, may play a significant role in the stress response associated with laparoscopic nephrectomy.

\section{ACKNOWLEDGMENTS}

This research was supported by the Department of Veterans Affairs and by United States Surgical Corporation.

\section{REFERENCES}

1. Kerbl K, Clayman RV, McDougall EM, et al. Transperitoneal nephrectomy for benign disease of the kidney: A comparison of laparoscopic and open surgical techniques. Urology 1994;43:607613.

2. Parra RO, Perez MG, Boullier JA, et al. Comparison between standard flank versus laparoscopic nephrectomy for benign renal disease. J Urol 1995;153:1171-1173.

3. Karayiannakis AJ, Makri GG, Mantzioka A, et al. Systemic stress response after laparoscopic or open cholecystectomy: A randomized trial. Br J Surg 1997;84:467-471.

4. Ortega AE, Peters JH, Incarbone R, et al. A prospective randomized comparison of the metabolic and stress hormonal responses of laparoscopic and open cholecystectomy. J Am Coll Surgeons 1996;183:249-256.

5. Bozkurt P, Kaya G, Altintas F, et al. Systemic stress response during operations for acute abdominal pain performed via laparoscopy or laparotomy in children. Anaesthesia 2000;55:5-9.

6. Yoshida S, Ohta J, Yamasaki K, et al. Effect of surgical stress on endogenous morphine and cytokine levels in the plasma after laparoscopic or open cholecystectomy. Surg Endosc 2000;14:137140.

7. Ellstrom M, Bengtsson A, Tylman M, et al. Evaluation of tissue trauma after laparoscopic and abdominal hysterectomy: Measurements of neutrophil activation and release of interleukin-6, cortisol, and C-reactive protein. J Am Coll Surgeons 1996;182:423-430.

8. Jakeways MSR, Mitchell V, Hashim IA, et al. Metabolic and inflammatory responses after open or laparoscopic cholecystectomy. Br J Surg 1994;81:127-131.

9. Wolf JS Jr, Moon TD, Nakada SY. Hand-assisted laparoscopic nephrectomy: Comparison to standard laparoscopic nephrectomy. J Urol 1998;160:22-27.

10. Bruhat MA, Goldschmit R. Minilaparoscopy in gynecology. Eur J Obstet Gynecol Reprod Biol 1998;76:207-210.

11. Dahl JB, Kehlet $H$. The value of pre-emptive analgesia in the treatment of postoperative pain. Br J Anaesth 1993;70:434-439.

12. Dasgupta P, Fowler CJ. Chilies: From antiquity to urology. $\underline{\mathrm{Br} J}$ Urol 1997;80:845-852.

13. Egdahl RH. Pituitary-adrenal response following trauma to the isolated leg. Surgery 1959;6:9-21.

14. Holton LL, Scott EM, Nolan AM, et al. Comparison of three methods used for assessment of pain in dogs. J Am Vet Med Assoc 1998;212:61-66.

15. Conzemius MG, Hill CM, Sammarco JL, et al. Correlation between subjective and objective measures used to determine severity of postoperative pain in dogs. J Am Vet Med Assoc 1997;210:1619-1622.

16. Desborough JP. The stress response to trauma and surgery. $\mathrm{Br} \mathrm{J}$ Anaesth 2000;85:109-117.

17. Ishizuka B, Kuribayashi K, Kobayashi Y, et al. Stress responses during laparoscopy with $\mathrm{CO}_{2}$ insufflation and with mechanical elevation of the abdominal wall. J Am Assoc Gynecol Laparoscopists 2000;7:363-371.

18. Gann DS, Foster AH. Endocrine and metabolic responses to injury. In: Schwartz SI (ed): Principles of Surgery. New York: McGrawHill, 1994, pp 4-9.

Address reprint requests to: Robert Marcovich, M.D. Dept. of Urology Long Island Jewish Medical Center 270-05 $76^{\text {th }}$ Ave. New Hyde Park, NY 11040-1496

E-mail: robertmarcovich@hotmail.com 
This article has been cited by:

1. Chad M. Devitt, Ray E. Cox, Jim J. Hailey. 2005. Duration, complications, stress, and pain of open ovariohysterectomy versus a simple method of laparoscopic-assisted ovariohysterectomy in dogs. Journal of the American Veterinary Medical Association 227:6, 921-927. [CrossRef]

2. David Canes, Veronica Triaca, Ingolf Tuerk. 2005. Laparoscopic radical cystectomy with continent urinary diversion. Current Urology Reports 6:2, 109-117. [CrossRef] 